\title{
OMAE2007-29061
}

\section{HYDRODYNAMIC AND THRUSTER MODEL VALIDATION FOR AUTONOMOUS UNDERWATER VEHICLES}

\author{
M. Chyba, T. Haberkorn, R.N. Smith; S.K. Choiłand Scott Weatherwax \\ *Department of Mathematics \\ 'Department of Ocean \& Resources Engineering \\ ¥Autonomous Systems Laboratory, The College of Engineering \\ University of Hawaii \\ Honolulu, Hawaii 96822 \\ Email:mchyba@math.hawaii.edu,haberkor@math.hawaii.edu, \\ ryan@ore.hawaii.edu,schoi@hawaii.edu,waxer@artifex.org
}

\begin{abstract}
From Pontryagin's Maximum Principle to the Duke Kahanamoku Aquatic Complex; we develop the theory and generate implementable time efficient trajectories for a testbed autonomous underwater vehicle (AUV). This paper is the beginning of the journey from theory to implementation. We begin by considering pure motion trajectories and move into a rectangular trajectory which is a concatenation of pure surge and pure sway. These trajectories are tested using our numerical model and demonstrated by our AUV in the pool. In this paper we demonstrate that the above motions are realizable through our method, and we gain confidence in our numerical model. We conclude that using our current techniques, implementation of time efficient trajectories is likely to succeed.
\end{abstract}

\section{Introduction}

In every area of research, merging theory and application is the ultimate goal, and is usually quite difficult. This

\footnotetext{
*This research is supported by NSF grants DMS-030641 and DMS-
} 0608583 difficulty arises especially in hydrodynamics and fluid mechanics due to the complexity of the flow and the dearth of a complete understanding of all the components involved. Our main research is focused on the implementation of time and/or energy efficient trajectories onto AUV's. This research involves developing a mathematical theory in optimal control, numerically calculating trajectories and implementing them onto a testbed AUV. The theory finds the extremals of the Hamiltonian system using Pontryagin's maximum principle. A numerical algorithm parameterizing the switching times of the thrusters has been developed which creates implementable trajectories which are close in time to the extremal trajectories found in the theory. These implementable trajectories are then tested on the OmniDirectional Intelligent Navigator (ODIN); a spherical vehicle with eight independent thrusters for control. Many previous implementations and experiments have yielded great success, but we are still interested in improving our results. Discrepencies between the experimental and theoretical trajectories can be related to many things such as actuator imperfections, inaccurate hydrodynamic model, unknown drift velocities and human error. Through a mul-

Copyright (c) 2007 by ASME 
titude of tests, we are able to see what parameters affect vehicle performance in what ways, and we can make adjustments in both the theory and the experiments in order to successfully and accurately implement efficient trajectories onto AUV's.

\section{Model}

The model we have chosen is driven by the test bed AUV that we use to test our theory. Any model is suitable, but for our purposes, we have chosen one that we can ground truth. We assume that we have a rigid body deeply submerged in a real fluid; we take real to be an ideal fluid with viscosity. It is understood in practice that a viscous fluid is rotational, but we assume this is not the case for theoretical reasons. We assume that the origin of the body fixed frame is the center of gravity $\left(C_{G}\right)$ of the body, the body has three planes of symmetry with body axes that correspond to the three principle axes of inertia. We do not take the assumption that the body is neutrally buoyant since our test-bed vehicle is slightly positively buoyant.

\section{Equations of Motion}

We consider the general equations of motion for marine vehicles in 6 degrees of freedom which stem from Newton's Second Law and express them as a controlled mechanical system. A detailed derivation can be found in [11]. We let $\eta=(x, y, z, \phi, \theta, \psi)^{t}$ be the position and orientation of the vehicle in the earth-fixed reference frame, where $(\phi, \theta, \psi)$ are the classical Euler angles. The translational and rotational resp. velocities $v=\left(v_{1}, v_{2}, v_{3}\right)^{t}$ and $\Omega=\left(\Omega_{1}, \Omega_{2}, \Omega_{3}\right)^{t}$ are taken with respect to the body-fixed frame. With this notation, we can write the equations of motion as,

$$
\begin{array}{r}
M \dot{v}=M v \times \Omega-D_{v}(v) v+F \\
J \dot{\Omega}=J \Omega \times \Omega+M v \times v-D_{\Omega}(\Omega) \Omega-r_{B} \times R^{t} \rho g \nabla k+\tau
\end{array}
$$

where $M$ accounts for the mass of the rigid body and the added mass coefficients, $J$ accounts for the body moments of inertia and the added moment of inertia coefficients and $\mathrm{F}$ and $\tau$ account for the control of the vehicle. The term $r_{B} \times R^{t} \rho g \nabla k$ is the restoring force which is a pure torque induced upon listing where $r_{B}$ is the vector from $C_{G}$ to the center of buoyancy $\left(C_{B}\right), \rho$ is the fluid density, $\mathrm{g}$ is the acceleration due to gravity, $\nabla$ is the submerged volume of the body and $k$ is the unit vector pointing in the direction of gravity.

\section{Test-Bed AUV}

The test-bed AUV which we use is the OmniDirectional Intelligent Navigator (ODIN) which is owned and operated by the Autonomous Systems Laboratory (ASL) at the University of Hawaii. As seen in Figure 1, ODIN has a spherical hull which is $65 \mathrm{~cm}$ in diameter.

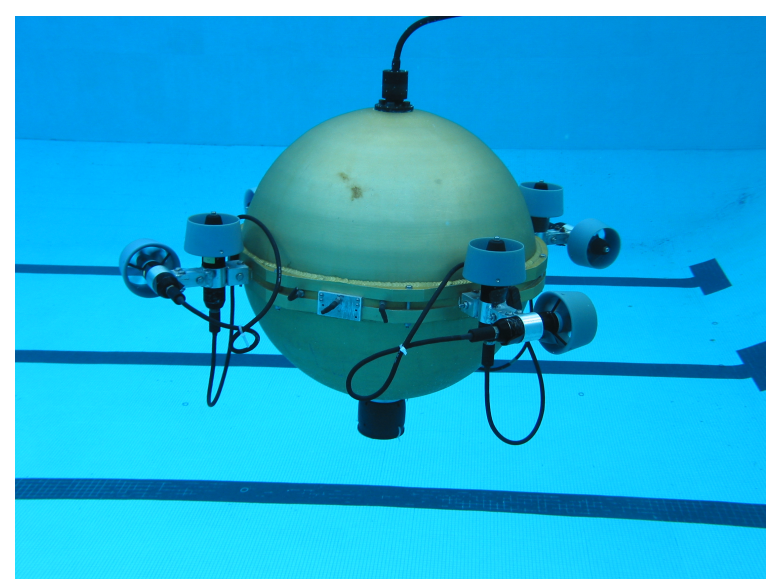

Figure 1. ODIN operating in the pool.

This sphere is constructed from an aluminum alloy to prevent corrosion. Eight thrusters are attached to the sphere via four fabricated mounts, each holding two thrusters. The thrusters are evenly distributed around the sphere with four vertical and four horizontal, see Figure 1. Fully assembled, ODIN weighs $126.55 \mathrm{~kg}$ and is positively buoyant by $0.7 \mathrm{~kg}$. ODIN is capable of moving in 6 DOF from either a remote or autonomous mode. For our experiments, ODIN is tethered, but only to send commands via TCP/IP protocol from a shore based laptop to be run in an autonomous mode. This setup allows for multiple tests to be conducted without removing ODIN from the water to upload mission sorties. ODIN's internal CPU is a $800 \mathrm{MHz}$ Intel based processor running on a $\mathrm{PC} 104+$ form factor with two external I/O boards providing A/D and D/A operations. Major internal components include a pressure sensor, internal navigation sensor and 24 batteries. ODIN is capable to compute real time, yaw, pitch, tilt, and depth and can run autonomously for up to 5 hours. The software is divided into two components. The first component is based 
on a real time extension to the Windows 2000 operating system, which provides ODIN real time autonomous control. The second component runs on the remote laptop and allows the operator to upload autonomous mission profiles to ODIN on the fly during testing as well as monitor ODIN in real time during such missions.

\section{Parameters}

The experiments conducted for this research are carried out at the Duke Kahanamoku Swimming Complex at the University of Hawaii. We use the testbed AUV ODIN. As noted above, ODIN does not have real time sensors to detect horizontal (x-y) position. Instead, experiments are video taped from the $10 \mathrm{~m}$ diving platform, giving us a near nadir view of ODIN's movements. Videos are saved and horizontal position is post processed for later analysis. A real time system utilizing sonar was available on ODIN, but was abandoned for two main reasons. First, the sonar created too much noise in the diving well and led to inaccuracies. More significantly, in the implementation of our optimal trajectories, ODIN is often required to achieve large $\left(>15^{\circ}\right)$ list angles which render the sonars useless for horizontal position. Many solutions were attempted and video led to the most accurate results.

Unique to ODIN's construction is the control from an eight dimensional thrust to move in 6 DOF. This construction puts redundancy into the system in case of thruster failure. ODIN is able to operate in an "underactuated" condition if necessary, and research is active in this area as well. Under certain circumstances of underactuation, ODIN is still fully controllable. Our input trajectories to ODIN take the form of the 6 DOF controls which are converted onboard ODIN to the 8 dimensional actual thrusters using the following Thrust Control Matrices (TCM's) (Eqns. 3 and 4).

TCMhorizontal $=$

$$
\left[\begin{array}{cccc}
-0.707 & 0.707 & 0.707 & -0.707 \\
0.707 & 0.707 & -0.707 & -0.707 \\
0.48160 & -0.48160 & 0.48160 & -0.48160
\end{array}\right]
$$

TCMvertical $=$

$$
\left[\begin{array}{cccc}
-1.0 & -1.0 & -1.0 & -1.0 \\
-0.26989 & -0.26989 & 0.26989 & 0.26989 \\
0.26989 & -0.26989 & -0.26989 & 0.26989
\end{array}\right]
$$

The numerical values of the various parameters used for the model are given in Table 1. These values were de- rived from experiments performed on ODIN. The added mass and drag terms were estimated from formulas found in $[12,13]$. Moments of inertia were calculated using experiments outlined in [14]. We used inclining experiments to locate and place $C_{G}$ in the location seen in Table 1 , and we assume that $C_{B}$ is located at the center of our body-fixed axis. Note that units for inertia coefficients (denoted by ${ }^{*}$ ) are $\mathrm{kg} \cdot \mathrm{m}^{2}$.

\begin{tabular}{|l|l||l|l||l|l|}
\hline$m$ & $126.55 \mathrm{~kg}$ & $\rho g \nabla$ & $1248 \mathrm{~N}$ & $C_{G}$ & $(0,0,-1) \mathrm{cm}$ \\
\hline$M_{f}^{u}$ & $70 \mathrm{~kg}$ & $M_{f}^{v}$ & $70 \mathrm{~kg}$ & $M_{f}^{w}$ & $70 \mathrm{~kg}$ \\
\hline$I_{x}$ & $5.46^{*}$ & $I_{y}$ & $5.29^{*}$ & $I_{z}$ & $5.72^{*}$ \\
\hline$J_{f}^{p}$ & $0^{*}$ & $J_{f}^{q}$ & $0^{*}$ & $J_{f}^{r}$ & $0^{*}$ \\
\hline$D_{v}^{11}$ & 35.789 & $D_{v}^{21}$ & 35.7953 & $D_{v}^{31}$ & 31.1602 \\
\hline$D_{v}^{12}$ & 36.836 & $D_{v}^{22}$ & 36.8312 & $D_{v}^{32}$ & 34.8251 \\
\hline$D_{\Omega}^{11}$ & 18.9379 & $D_{\Omega}^{21}$ & 18.9379 & $D_{\Omega}^{31}$ & 16.8545 \\
\hline$D_{\Omega}^{12}$ & 35.4235 & $D_{\Omega}^{22}$ & 34.4235 & $D_{\Omega}^{32}$ & 31.5264 \\
\hline
\end{tabular}

Table 1. Numerical values for hydrodynamic coefficients.

Along with the tests to determine the values in Table 1, we also tested the thrusters. Each thruster has a unique voltage input to power output relationship. This relationship is highly nonlinear and is approximated using a piecewise linear function which we refer to as our thruster model. Hence, we still see residual "phantom" thrusts occurring due to the unbalancing or non-synchronization of the eight thrusters. The most detrimental "phantom" moment we encounter is in the yaw direction, partially due to the lack of a restoring moment along with small drag resistance.

\section{Experiments}

Below we analyze two experiments of pure motion trajectories implemented on ODIN. The first is a pure heave, and the second a pure surge. In each case, we analyze three separate trajectories in order to help understand the model and dynamics. The first trajectory is the experimental trajectory which is the actual trajectory realized by ODIN in 
the pool. This is plotted using the data collected directly from ODIN's sensors and the video camera for orientation, depth and position. The second trajectory analyzed is the pre-expeminent theoretical trajectory, or pre-theoretical trajectory. This is the path that the computer model says that ODIN should follow with the given theory and hydrodynamic model. The third is a combination of the first and second aforementioned in that we input the actual voltage that ODIN applied to the thrusters. We will refer to this trajectory as the post experiment theoretical trajectory, or post-theoretical trajectory. The post-theoretical trajectory is of importance because it highlights inaccuracies we may have in our model that are not apparent by comparing just the pre-theoretical and experimental trajectories. In the experimental trajectories, we have smoothed the depth data due to sensor noise.

\section{Pure Heave}

For this experiment, we conducted a closed loop dive to approximately $1.5 \mathrm{~m}$ and stabilized the vehicle there. This stabilization is an initialization sequence which starts every experiment. We use $1.5 \mathrm{~m}$ since this is roughly 2.5 diameters of our vehicle and thus we do not have to consider Froude-Krylov forces or wave damping forces since we are "deeply submerged"; yet at $1.5 \mathrm{~m}$ the vehicle is still easily accessible underwater and allows for plenty of depth below it with which to run experiments. When the vehicle was stabilized (ie. no obvious visual motions) we then prescribed a $2.3 \mathrm{~m}$ pure dive in open loop with feedback control only in pitch and roll. The results are shown in Figure 2 where the solid line is the experimental trajectory, the dotted line is the pre-theoretical trajectory and the dashed line is the post-theoretical trajectory. Note that the legend is the same for all subsequent graphs.In the figures, we see that we applied a $30 \mathrm{~N}$ force in the z-axis for approximately 5 seconds. The experimental trajectory deviates from the two theoretical by undershooting the prescribed depth, and then rising too quickly after completion of the dive. This is believed to be caused by an underestimate of the drag coefficient in the heave direction and inaccuracies in the thruster model. In particular, we may have prescribed a $30 \mathrm{~N}$ thrust, but we may have gotten more or less of a thrust. Note that the roll and pitch are not steady which is due mainly to the thruster model, but both are within $2^{\circ}$ which is not noticeable when viewing the experiment.
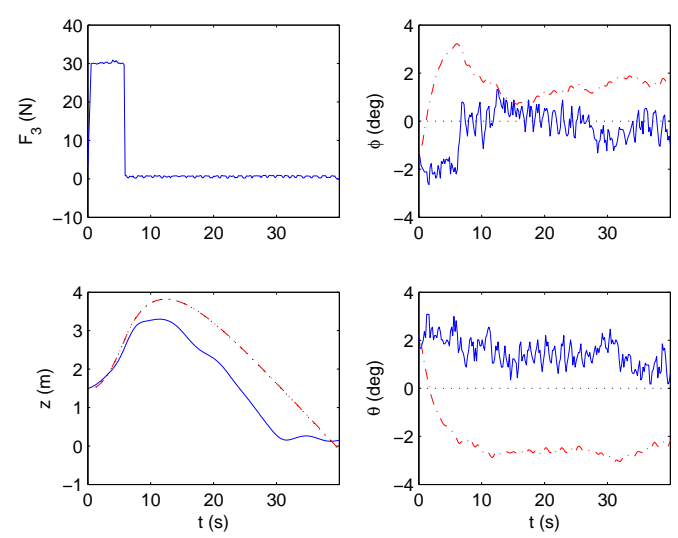

Figure 2. Pure Heave Motion of $2.3 \mathrm{~m}$.

\section{Pure Surge}

For this experiment, after initial stabilization, we prescribed a $4 \mathrm{~m}$ pure surge in the negative direction. The trajectory was applied in open loop with only a yaw feedback loop running. This is necessary due to the current in the pool from the recirculation, and the slight differences in thrusters mentioned above. Since there is no restoring force in the yaw direction, without a feedback loop, a straight line is nearly impossible.
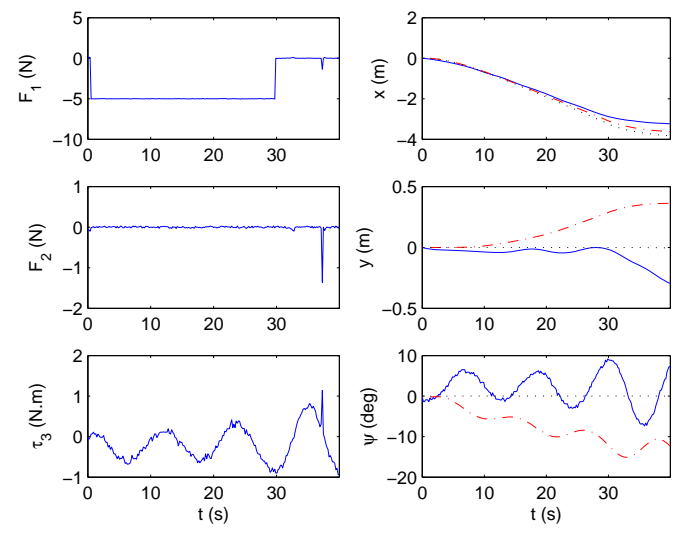

Figure 3. Pure Surge Motion.

In the $\mathrm{x}$-direction, we see that all three trajectories are very similar and that we end up about $1 \mathrm{~m}$ short in the experimental trajectory. This is most likely the result of an underestimate of the drag coefficient in the surge (sway by symmetry). The y-direction shows a deviation even though 
we did not prescribe any at all. This is due to the yaw controller adjusting for the thruster inconsistencies and slightly by the drift in the pool. By looking at the $\tau_{3}$ component, we note that we are applying less than $1 \mathrm{~N}$ thrust for a yaw compensation of $10^{\circ}$.

\section{Concatenation}

Now, we are interested in our ability to combine the above pure motions. In order to test this, we prescribe the trajectory of a rectangle with base $4 \mathrm{~m}$ and height $3 \mathrm{~m}$ as our goal trajectory. To achieve this motion, we consider two techniques. First, through the use of ODIN we are very familiar with her operation, and know that given an applied thrust of $15 \mathrm{~N}$ in surge for 15 seconds, ODIN moves about $4 \mathrm{~m}$. Likewise for the same thrust applied for 10 seconds, ODIN moves about $3 \mathrm{~m}$. Now, remembering that we are in an unbounded real fluid, we create our rectangle trajectory by applying $F_{1}=13 \mathrm{~N}$ for $15 \mathrm{sec} ., F_{2}=13 \mathrm{~N}$ for 12 sec., $F_{1}=-13 \mathrm{~N}$ for $15 \mathrm{sec}$., $F_{2}=-13 \mathrm{~N}$ for $12 \mathrm{sec}$. At the corners and the end of the trajectory, we give $3 \mathrm{sec}-$ onds of no applied forces to ensure that ODIN has come to a complete stop before beginning the next leg. This thrust strategy is run through our numerical model to produce the pre-theoretical trajectory, which most times is not a perfect rectangle. This is a purely ad hoc method, yet we achieved descent results in many experiments. An example is shown in Figure 4.

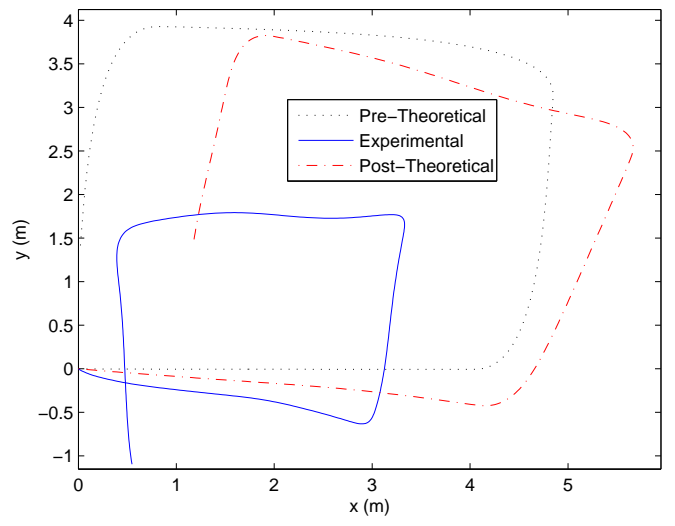

Figure 4. Ad Hoc Rectangle Trajectories.

ODIN clearly completed the rectangle, but made a scaled down version, and ended up about $1 \mathrm{~m}$ from where she should have. The post-theoretical trajectory matches the pre-theoretical quite well if you account for a yaw offset at the beginning of each leg. Although ODIN follows a straight line, it may not be in the prescribed direction. This aspect is under investigation, but as above, the angular displacement along each leg is on the order of $15^{\circ}$ which can be obtained with as little as 1 N.m of $\tau_{3}$ thrust.

Another technique is to insist that the vehicle comes to rest at each corner to stabalize before attempting the next leg of the rectangle. This type of trajectory takes longer to realize since we apply a deceleration phase into each leg of the rectangle and stabilize ODIN longer at each corner, but it creates a better rectangle than the earlier described technique. Here we actually figure out the exact thrust that our model predicts to move a given distance with the assumption that the initial and final velocity of the vehicle is zero. We will analyze this experiment a bit closer.

To begin, we conducted the same initialization as previous, and then prescribed a $5 \mathrm{~m}$ by $4 \mathrm{~m}$ rectangular motion seen in Figure 5. First note that each trajectory is a rectangle with nice sharp corners. Again ODIN creates a scaled down rectangle from that prescribed. Note that at the first two corners there are loops in the trajectory, this further emphasizes the underestimation of the drag in surge and sway since the vehicle actually moved backward during its deceleration phase when we predicted it to have zero velocity at the end of the phase. Also, we see again the post-theoretical trajectories is more or less straight and only differs from the pre-theoretical trajectory by an angular disturbance along each leg. Further investigation will reveal the effect of the drift in the pool, which was first thought to be negligible.

\section{CONCLUSIONS}

In this paper we analyze the implementation of open loop trajectories onto a test bed AUV. We consider two pure motion trajectories and analyze the implementation and the model used for computation and theoretical modeling. We have found that our angular disturbances are minimal, with the most problem coming from the yaw direction. We have been able to show that our ability to follow pure motion trajectories is excellent even with a feedback controller only working in yaw. This is a direct relation to producing a good model for our vehicle. These experiments lead us to investigate the drag relationship more closely. It seems that we need to slightly increase the drag coefficient of our ve- 


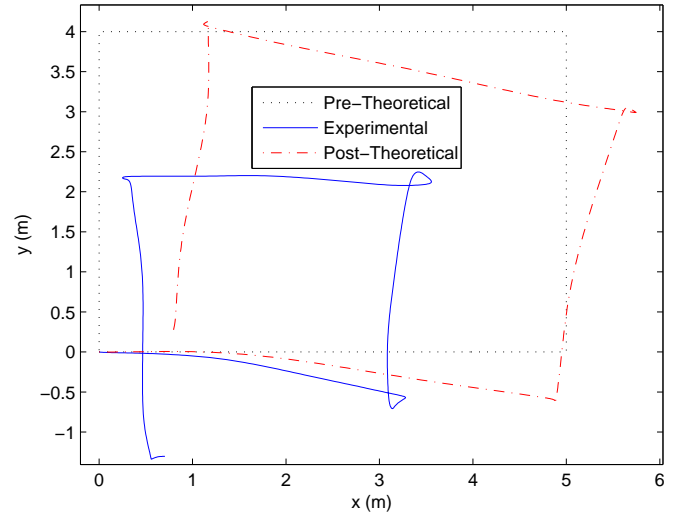

Figure 5. Calculated Rectangular Trajectories.

hicle. More importantly is the need for an accurate model for our thrusters. Since ODIN's design is for controllability rather than stability, and we lack resistance and restoring forces in yaw, small differences in the thrusters causes noticeable motions during experiments. We are currently running tests to determine more accurate estimates on the thruster models, which will also give insight as to where our drag estimates need adjustment. Along with this, we also see a need to implement a consolation for the current in the pool which was first assumed to be negligible. We have a descent Lagrangian understanding of the pool drift, but are assessing methods to provide an Eulerian approach which can be easily implemented into our model. With a few minor adjustments, we will achieve an accurate rectangle with both the post-theoretical and experimental trajectories. After this success, we plan to begin implementation and tracking of time optimal trajectories. These trajectories can be quite complex and involve a superposition of many if not all degrees of freedom in each movement. Thus, it is important to validate all models on simple concatenations to trust the results extracted from later experiments.

\section{REFERENCES}

[1] M. Chyba, N.E. Leonard and E.D. Sontag. "Singular trajectories in the multi-input time-optimal problem: Application to controlled mechanical systems". Journal on Dynamical and Control Systems 9(1):73-88, 2003.

[2] M. Chyba, N.E. Leonard and E.D. Sontag. "Optimality for underwater vehicles". In Proceedings of the 40th IEEE Conf. on Decision and Control, Orlando, 2001.

[3] M.Chyba, H. Maurer, H.J. Sussmann and G. Vossen. "Underwater Vehicles: The Minimum Time Problem". In Proceedings of the 43th IEEE Conf. on Decision and Control, Bahamas, 2004.

[4] M. Chyba and T. Haberkorn. "Designing efficient trajectories for underwater vehicles using geometric control theory". Proceedings of the 24rd International Conference on Offshore Mechanics and Arctic Engineering, Greece, 12-17 June 2005.

[5] B. Bonnard and M. Chyba. "Singular Trajectories and their Role in Control Theory". Springer-Verlag, 2003.

[6] M. Chyba and T. Haberkorn. "Autonomous underwater vehicles: singular extremals and chattering". Proceedings of the 22nd IFIP conference on System modeling and Optimization, Turin, Italy, 18-22 July 2005. To appear.

[7] R. Fourer, D.M. Gay and B.W. Kernighan. "AMPL: A Modeling Language for Mathematical Programming". Duxbury Press, Brooks-Cole Publishing Company, 1993.

[8] A. Waechter and L. T. Biegler. "On the Implementation of an Interior-Point Filter-Line Search Algorithm for Large-Scale Nonlinear Programming". Research Report RC 23149, IBM T.J. Watson Research Center, Yorktown, New-York.

[9] C.Y. Kaya and J.L. Noakes. "Computation method for time-optimal switching control". Journal of $\mathrm{Op}$ timization Theory and Applications, 117(1):69-92, 2003.

[10] H. Maurer, C. Büskens, J-H.R. Kim and I.C. Kaya. "Optimization methods for numerical verification of second order sufficient conditions for bang-bang controls". Optimal Control Application and Methods, 26:129-156, 2005.

[11] M. Chyba, T. Haberkorn, R.N. Smith and G.R. Wilkens. "Design of Time Efficient Trajectories for a Submerged Rigid Body". ASK MONIQUE.

[12] E. Eugene Allmendinger. "Submersible Vehicle Design”. SNAME, 1990.

[13] F.H. Imlay. "The Complete Expressions for Added Mass of a Rigid Body Moving in an Ideal Fluid". Technical Report DTMB 1528, 1961.

[14] Rameswar Bhattacharyya. "Dynamics of Marine Vehicles". Wiley, 1978. 